\title{
Case Study: EIMS Acupuncture Treatment for Posterior Tibialis Tendonitis
}

\author{
N. Yelizarov'1, J. Taunton2,3* \\ ${ }^{1}$ Muscle \& Tendon Clinic, Richmond, Canada \\ ${ }^{2}$ Division of Sports Medicine, Faculty of Medicine, University of British Columbia, Vancouver, Canada \\ ${ }^{3}$ Sports Medicine Fortius Sport \& Health, Vancouver, Canada \\ Email:nikyeliz@gmail.com, ${ }^{*}$ jack.taunton@ubc.ca
}

Received 7 August 2015; accepted 6 November 2015; published 9 November 2015

Copyright (C) 2015 by authors and Scientific Research Publishing Inc.

This work is licensed under the Creative Commons Attribution International License (CC BY). http://creativecommons.org/licenses/by/4.0/

cC) (i) Open Access

\begin{abstract}
BACKGROUND AND OBJECTIVE: Posterior tibialis tendon dysfunction (PTTD) is a progressive ailment of a lower limb that, if left untreated, can lead to irreversible changes in the structure and function. The authors of the present study hypothesized the damages to the tibias tendon can be countered and reversed using electrical acupuncture and concurrent treatment of the associated muscle. INTERVENTION: The following study details a successful treatment of the early stage PTTD in a group of competitive athletes using a specific Electrical Intra Muscular Stimulation (EIMS) protocol developed and rooted in years of practice and the knowledge of the pathophysiology of the muscle and tendon tissues. Specifically, a combination the posterior and the original EIMS approach was used to stimulate the tendon and muscle concurrently at a frequency of $2 \mathrm{~Hz}$. Analysis of the VAS scores showed a significant improvement following the treatment sessions. CONCLUSION: The success of this combined muscle/tendon stimulation protocol is given a strong correlation between the mechanism of tendon and muscle injury and recovery. The fast rate of healing and the simplicity of the materials required for the treatment offer a great potential for a wide-spread application. The authors extrapolate that the treatment method, with applicable modifications in needling locations, can be used for an array of common ailments involving tendons and muscles of the lower limb and foot, including the over-pronation, which is now common place due to the overweight problem in the general population.
\end{abstract}

\section{Keywords}

Tibialis Posterior Tendonitis, EIMS, Repetitive, Muscle Strain, Competitive Diving

"Corresponding author. 


\section{Introduction}

Posterior tibialis tendon dysfunction (PTTD) is a common cause of foot pain and dysfunction in adults [1] [2]. About $10 \%$ of the general population is affected by this impairment. Clinical observations strongly suggest that the condition is progressive [2]. Descriptively, the varying presentations of this condition are divided into four stages. Stage I is characterized by mild swelling and medial ankle pain but no deformity. Stage II is characterized by progressive flattening of the arch, with an abducted mid-foot. In stage IIA, the foot is still flexible; however, the tendon is functionally impaired. In stage IIB, the tendon is incompetent, or even ruptured. Stage III includes all of the signs of stage II; however, the hind foot deformity becomes fixed. The physiologic and pathologic changes occur within the tendon as a result of microtears, inflammation and rupture that occurred as a consequence of repetitive loading [3]. Stage IV dysfunction, as described by Myerson, occurs when degenerative changes are also present in the ankle joint. Despite its high prevalence [1], there are no intervention guidelines for Stage I or II PTTD. Surgical repair is the only definitive treatment for Stage III or IV [3].

We searched Pub Med for publications using the keywords "tibialis posterior" and "acupuncture" and only two relevant articles came up. The most relevant to the study is an article [4] describing a case of one triathlete, who was treated with a combination of protocols one of which was electrical stimulation with needles and it was referred to as "medical acupuncture". The second article [5] focused on the depth and direction of needling of tibialis posterior muscle through at Zusanli (ST 36), proximal 1/3 of tibia with lateral approach, which in our opinion is not advantageous mainly due to a difficulty to place the second needle (or to fail-proof to properly locate the muscle). This study was performed on 80 subjects and the results weren't reported. Our study was done on four athletes competing at the international level.

EIMS has been used in Richmond Muscle \& Tendon Clinic for the last 15 years with consistent improvement in different types of soft tissue injuries among a broad spectrum of patients. However, it was noted that competitive athletes with tibialis posterior responded differently to a standard treatment protocol when compared to a general pool of patients. The current study of athletes with tibialis posterior tendinitis was motivated by the observed lack of recovery progress in an elite athlete in July 2012 following the treatment that was found effective for a general public in the same period of time.

The research questions were:

1) What are the optimal EIMS parameters for a successful treatment of PTT in competitive athletes?

2) Is the improvement in health outcomes sustained and consistent between the subjects?

\section{Method}

\subsection{Design}

\subsubsection{Visual Analogue Scale for Pain (VAS)}

The VAS is a single dimension scale with endpoints marked as "no pain" and "worst pain possible". It is a reliable and valid measure of self-reported pain intensity. The VAS was used to measure pain/discomfort intensity during regular day of training activity of each involved subject.

\subsubsection{Study Limitations}

Size of the study population;

Type of athletic activity;

Long-term follow-up data not available

The study did not apply to such injuries as acute PTT, contusions, lacerations, abrasions or injuries due to a fall or an accident as well as PTT injuries out of time frame 1 - 8 months of existing symptoms.

\subsubsection{Method Limitations}

1) For reporting of the outcome, VAS is used. This is a subjective measurement tool, essentially self-reporting the condition of one's pain or discomfort in a specific area. Our treatment target was specifically PTT. But if patients have a number of ailments in the same part of the anatomy, their VAS reporting may become compounded by the worsening or improvement in the status of other unrelated conditions.

2) The power analysis of the study is to be based on differences that are assumed and not directly determined through the literature. 
3) The difference in "Before” and "After" was based on VAS scores only, which is subjective but no other option was available at the time.

\subsubsection{Timing of Evaluation}

Each subject was evaluated with VAS before and after intervention at the same time points as other subjects to control for and minimize any possible variations in the outcome.

55 days follow-up

Two subjects were re-evaluated 55 days after the final treatment session using the same VAS.

\subsection{Participants, Therapists, Centers}

The athletes enrolled in the study were Caucasian, 1 male and 3 female. Female ages were 15, 16 and 24 and male age was 22. All subjects had a high lean body mass ratio. All participants were competitive high platform and springboard divers. Their training regimen was rigorous, typically clocking 8 hours a day. The described treatment intervention was provided during the active training every day, typically after 6 pm when the training was over for the day.

\subsection{Intervention}

Each subject was needled with two needles, whereby one was inserted into PTT at the lower $1 / 3$ of tibia and the second one was inserted into Posterior Tibialis muscle (Figure 1) using posterior approach [6] which comprises the needling at the lateral $1 / 3$ of calf's width of proximal $1 / 3$ of tibia length.

The stainless disposable acupuncture needles $0.3 \times 50 \mathrm{~mm}$ were connected through alligators and wires to electro-acupuncture device AWQ104E (Figure 2) and electrical stimulation was applied for $30-45$ min. The parameters were a fixed frequency of $2 \mathrm{~Hz}$ and the criteria for sufficient intensity were noticeable muscle contractions within a comfort zone of each subject. The procedure was repeated every day for one week.

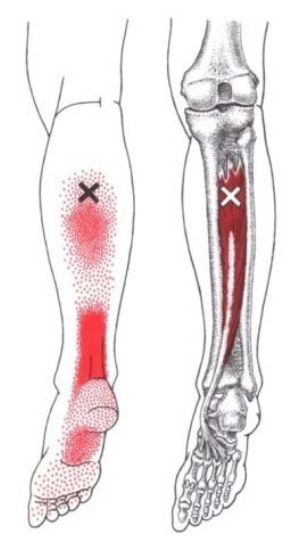

Figure 1. The point of needle insertion into the posterior tibialis muscle.

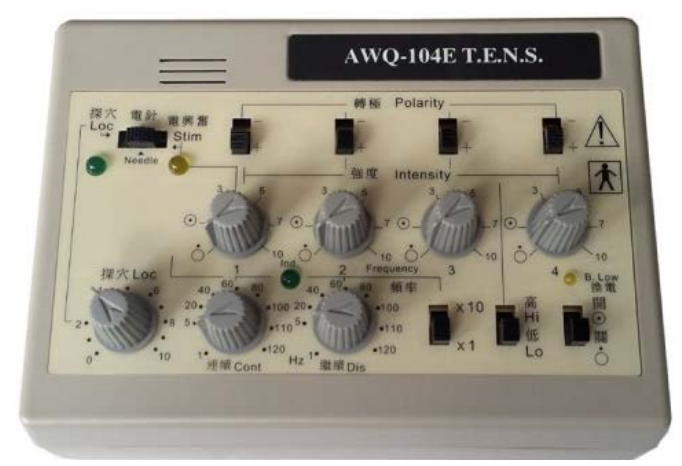

Figure 2. The electro-acupuncture device AWQ104E used in the study. 
The design of this study was based on a typical EIMS treatment with the needling of affected muscle/tendon with gentle low frequency electrical stimulation such of $2 \mathrm{~Hz}$ and intensity determined by visible muscle twitching. A tendon injury requires a dedicated approach that is based on needling of muscle and tendon which are then connected to the same wire and electrical stimulation is applied with the same frequency but less intensely.

\subsection{Outcome Measures}

Primary outcome: decrease in pain and swelling in posterior tibia tendon

Secondary outcome: gain of normal athletic performance

\subsection{Data Analysis}

Two types of statistical design were used to explore the difference in subjects before and after intervention T-test: (Table 1) and repeated measures ANOVA (Table 2). An artificial data were created to make possible ANOVA design. The data was calculated as mean of VAS score at 2 time points (Before and After) and labeled as Middle. The used statistical software was SPSS 13.0 version.

A paired samples t-test was conducted to evaluate impact of treatment on 3 subjects' VAS scores. There was a significant difference between Before $(M=90, S D=0.00)$ and After $(M=13.3, S D=5.77)$ in VAS scores; $\mathrm{t}(2)$ $=23, \mathrm{p}<0.002$. The eta squared statistic (0.99) indicated a large effect size.

Repeated ANOVA (Table 2)

There was a significant effect on VAS (Wilk's Lambda $=0.004, F(1,2)=529, p<0.05$, multivariate partial eta squared $=0.996$ ) with a large effect size.

\section{Results}

All subjects reported positive results (decrease in VAS scores) and a subjectively improved performance after six EIMS treatment sessions.

\section{Discussion}

Competitive divers put an excessive strain on posterior tibialis muscle in the course of their performance and training routine, accounting for the eventual tendinopathy (Figure 3). There are several key reasons for developing tendinopathy. One of them is a combination of mechanical overloading of the tendon and a diminished blood flow [7]. Excessive mechanical loading with or without the presence of intrinsic factors is contributing to the development of tendinopathy. While an etiology of the process in unclear, ischemia during a load or hypoxia in general may cause the damage resulted in tendinopathy [8]. Therefore treatment targeting a tendon's ischemia

Table 1. The difference in subjects before and after intervention: T-test analysis.

\begin{tabular}{|c|c|c|c|c|c|c|c|c|}
\hline \multicolumn{9}{|c|}{ Paired Sample Test } \\
\hline & \multicolumn{5}{|c|}{ Paired Differences } & \multirow{3}{*}{$\mathrm{t}$} & \multirow{3}{*}{$\mathrm{df}$} & \multirow{3}{*}{ Sig. (2-tailed) } \\
\hline & \multirow[t]{2}{*}{ Mean } & \multirow[t]{2}{*}{ Std. Deviation } & \multirow[t]{2}{*}{ Std. Eorror Mean } & \multicolumn{2}{|c|}{$\begin{array}{l}\text { 95\% Confidence Interval } \\
\text { of the Difference }\end{array}$} & & & \\
\hline & & & & Lower & Upper & & & \\
\hline Paire 1 Before - After & 76.67 & 5.774 & 3.333 & 62.324 & 91.009 & 23.000 & 2 & 0.002 \\
\hline
\end{tabular}

Table 2. The difference in subjects before and after intervention: repeated measures ANOVA.

\begin{tabular}{ccccccc}
\hline & \multicolumn{3}{c}{ Multivariate Tests $^{\mathrm{b}}$} \\
\hline Effect & Value & F & Hypothesis df & Error df & Sig. & Partial Eta Squared \\
\hline factor 1 Pillai's Trace & 0.996 & $529.000^{\mathrm{a}}$ & 1.000 & 2.000 & 0.002 & 0.996 \\
Wilks' Lambda & 0.004 & $529.000^{\mathrm{a}}$ & 1.000 & 2.000 & 0.002 & 0.996 \\
Hotelling's Tace & 264.500 & $529.000^{\mathrm{a}}$ & 1.000 & 2.000 & 0.002 \\
Roy's Largest Root & 264.500 & $529.000^{\mathrm{a}}$ & 1.000 & 2.000 & 0.002 & 0.996 \\
\hline
\end{tabular}

${ }^{\mathrm{a}}$ Exact statictic; ${ }^{\mathrm{b}}$ Design: Intercept within subjects design: factor 1. 


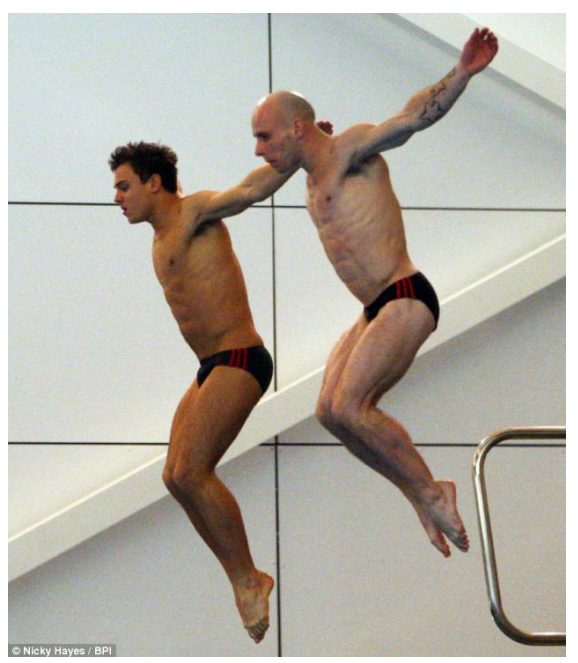

Figure 3. Example of the "heels up” position frequently assumed in competitive diving.

or hypoxia may enhance rehabilitation of tendinopathy. Tendon's blood supply comes from three major sources: myotendinous junction, osteotendinous junction, and paratenon [8]. Considering that myotendinous junction is the major one and that tendon receives about $1 / 3$ of muscle's blood circulation, a compromised muscle blood circulation will affect tendon's blood supply and its ability to heal. Given that a tendon injury has a strong correlation with a muscle belly injury [9], it is safe to conclude that tendon injury should be treated concurrently with the treatment of the corresponding muscle and the treatment should be focused on the rehabilitation of muscle strength and flexibility rather than on simply suppressing the inflammatory reaction.

There are articles that confirm a positive correlation between an increased blood flow and the EIMS/PENS when the treatment is applied with a specific frequency [10]. Also there are a number of articles supporting supremacy of PENS over TENS [11] [12].

The tested rear approach EIMS needling of treating tibialis posterior seems to be a very attractive therapeutic alternative for athletes mainly because of the quick improvement results without interrupting a training schedule, while being doping-safe and serving as a preventative measure for a serious injury of tibialis posterior. Due to a much greater routine wear and tear of the muscle and tendon tissue in training athletes, this segment of population is notoriously more resistant to treatment than general population. Hence, a positive treatment outcome in athletes can be extrapolated as having an even greater potential for an overall population. As mentioned afore, the incidence of the PTT problem is high, especially amongst the athletes, whereby in a relatively small group of divers (approx. 20 - 25) 4 had posterior tibial tendinopathy.

The results of the study should be transferred to the everyday practice for treating patients with over-pronated foot or valgus feet, which can be inborn or acquired. There are articles suggesting that tibialis posterior injury is typical for overweight patients [13] because of the correlation between the excess of the body weight and feet pronation [3]. Considering a growing problem of overweight among Canadian population and the difficulty of delivering an adequate solution for pronated feet in athletes [4] and general population [1], the current EIMS study may provide a cost-effective alternative and a conservative, sustainable approach to rectifying this impairment.

\section{Ethics Approval}

The General Ethics Committee(s) approved this study. All participants gave written informed consent before data collection began.

\section{References}

[1] Kohls-Gatzoulis, J., Angel, J.C., Singh, D., Haddad, F., Livingstone, J. and Berry, G. (2004) Tibialis Posterior Dysfunction: A Common and Treatable Cause of Adult Acquired Flatfoot. BMJ, 329, 1328-1333. http://dx.doi.org/10.1136/bmj.329.7478.1328 
[2] Durrant, B., Chockalingam, N. and Hashmi, F. (2011) Posterior Tibial Tendon Dysfunction: A Review. Journal of the American Podiatric Medical Association, 101, 176-186. http://dx.doi.org/10.7547/1010176

[3] Mendicino, S.S. (2000) Posterior Tibial Tendon Dysfunction. Diagnosis, Evaluation, and Treatment. Clinics in Podiatric Medicine and Surgery, 17, 33-54, vi.

[4] Howitt, S., Jung, S. and Hammonds, N. (2009) Conservative Treatment of a Tibialis Posterior Strain in a Novice Triathlete: A Case Report. The Journal of the Canadian Chiropractic Association, 53, 23-31.

[5] Lou, X.F., Yang, X.D., Jiang, S.H., Sun, C.Y. and Zhang, R.F. (2006) Study on Angle and Depth of Needle Insertion in Acupuncture at Zusanli (ST 36). Zhongguo zhen jiu = Chinese Acupuncture \& Moxibustion, 26, 483-486

[6] Rha, D.W., Im, S.H., Lee, S.C. and Kim, S.K. (2010) Needle Insertion into the Tibialis Posterior: Ultrasonographic Evaluation of an Anterior Approach. Archives of Physical Medicine and Rehabilitation, 91, 283-287. http://dx.doi.org/10.1016/j.apmr.2009.09.024

[7] Astrom, M. (2000) Laser Doppler Flowmetry in the Assessment of Tendon Blood Flow. Scandinavian Journal of Medicine and Science in Sports, 10, 365-367. http://dx.doi.org/10.1034/j.1600-0838.2000.010006365.x

[8] Sharma, P. and Maffulli, N. (2005) Tendon Injury and Tendinopathy: Healing and Repair. The Journal of Bone \& Joint Surgery Am, 87, 187-202. http://dx.doi.org/10.2106/JBJS.D.01850

[9] Hasselman, C.T., Best, T.M., Seaber, A.V. and Garrett Jr., W.E. (1995) A Threshold and Continuum of Injury during Active Stretch of Rabbit Skeletal Muscle. The American Journal of Sports Medicine, 23, 65-73. http://dx.doi.org/10.1177/036354659502300111

[10] Sandberg, M.L., Sandberg, M.K. and Dahl, J. (2007) Blood Flow Changes in the Trapezius Muscle and Overlying Skin Following Transcutaneous Electrical Nerve Stimulation. Physical Therapy, 87, 1047-1055. http://dx.doi.org/10.2522/ptj.20060178

[11] Yokoyama, M., Sun, X., Oku, S., et al. (2004) Comparison of Percutaneous Electrical Nerve Stimulation with Transcutaneous Electrical Nerve Stimulation for Long-Term Pain Relief in Patients with Chronic Low Back Pain. Anesthesia and Analgesia, 98, 1552-1556. http://dx.doi.org/10.1213/01.ANE.0000112312.94043.DF

[12] Weiner, D.K., Rudy, T.E., Glick, R.M., et al. (2003) Efficacy of Percutaneous Electrical Nerve Stimulation for the Treatment of Chronic Low Back Pain in Older Adults. Journal of the American Geriatrics Society, 51, 599-608. http://dx.doi.org/10.1034/j.1600-0579.2003.00202.x

[13] Lhoste-Trouilloud, A. (2012) The Tibialis Posterior Tendon. Journal of Ultrasound, 15, 2-6. http://dx.doi.org/10.1016/j.jus.2012.02.001

\section{Abbreviations}

EIMS: electrical intramuscular stimulation; PTTD: Posterior tibialis tendon dysfunction; VAS: visual analogue scale. 